\title{
Scaling at chiral quantum critical points in two dimensions
}

\author{
L. Schweitzer ${ }^{1}$ and P. Markošs \\ ${ }^{1}$ Physikalisch-Technische Bundesanstalt (PTB), Bundesallee 100, 38116 Braunschweig, Germany \\ ${ }^{2}$ Institute of Nuclear and Physical Engineering, FEI, \\ Slovak University of Technology, 81219 Bratislava, Slovakia
}

\begin{abstract}
We study the localization properties of electrons moving on two-dimensional bi-partite lattices in the presence of disorder. The models investigated exhibit a chiral symmetry and belong to the chiral orthogonal (chO), chiral symplectic (chS) or chiral unitary (chU) symmetry class. The disorder is introduced via real random hopping terms for chO and chS, while complex random phases generate the disorder in the chiral unitary model. In the latter case an additional spatially constant, perpendicular magnetic field is also applied. Using a transfer-matrix-method, we numerically calculate the smallest Lyapunov exponents that are related to the localization length of the electronic eigenstates. From a finite-size scaling analysis, the logarithmic divergence of the localization length at the quantum critical point at $E=0$ is obtained. We always find for the critical exponent $\kappa$, which governs the energy dependence of the divergence, a value close to $2 / 3$. This result differs from the exponent $\kappa=1 / 2$ found previously for a chiral unitary model in the absence of a constant magnetic field. We argue that a strong constant magnetic field changes the exponent $\kappa$ within the chiral unitary symmetry class by effectively restoring particle-hole symmetry even though a magnetic field induced transition from the ballistic to the diffusive regime cannot be fully excluded.
\end{abstract}

PACS numbers: 71.23.An,73.20.Fz,72.80.Vp

\section{INTRODUCTION}

The properties of electrons moving in disordered twodimensional (2D) lattices featuring special symmetries represent a long-standing challenge for theories of Anderson localization $\underline{1}^{\underline{1}}$ It is known that the basic mechanism for electron localization, i.e., quantum interference of an electron moving along different paths in disordered media, depends on the symmetry and topology of the particular model ${ }^{2,3}$ Recently, the discovery of topological insulators $\underline{\underline{4}}$ and the utilization of graphene $\mathrm{E}^{5,6}$ two-dimensional honeycomb lattice of carbon atomshas been producing interest in this topic anew. In the presence of disorder that preserves the sublattice symmetry of the underlying two equivalent interpenetrating triangular sublattices, the energy eigenvalues still come in pairs $\pm \epsilon_{i}$ around the Dirac point at $E=0$. The phenomenon of a quantum critical point at zero energy is not linked to graphene's hexagonal lattice but can arise also in other bi-partite lattices like the simple square lattice. Disordered systems possessing this property belong to the chiral symmetry classes $\frac{7,8}{1.8}$ One distinguishes three chiral Gaussian random matrix ensembles: the chiral orthogonal $(\mathrm{ChO})$, the chiral unitary $(\mathrm{ChU})$, and the chiral symplectic $(\mathrm{ChS})$ one, which differ from each other by the presence $(\mathrm{ChO})$ or absence $(\mathrm{ChU})$ of time reversal symmetry. The chS ensemble has time reversal symmetry but spin-rotational symmetry is lacking, due to spin-orbit interactions in most cases. An additional particle-hole symmetry is obeyed in the chO and chS classes.

In disordered 2D chiral systems, all electronic states are localized with an energy dependent localization length that diverges at the band center $E=0 . \underline{\underline{9}} \underline{\underline{13}}$ The predicted divergence of the disorder averaged density of states (DOS) obtained from non-linear sigma-model field theories $14-18$ was apparently observed in numerical studies, $\frac{19}{1}$ although the precise value of the critical exponent remained unclear. On the contrary, in numerical investigations ${ }^{20}$ of Motrunich et al. for chiral orthogonal systems (time-reversal symmetry is conserved), the exponent $\kappa$ of the asymptotic energy dependence of the disorder averaged DOS, $\rho(E) \sim E^{-1} \exp \left(-c|\ln E|^{\kappa}\right)$ was found to be compatible with $\kappa=2 / 3$, where $c$ is a nonuniversal constant. This result is at variance with the predicted universal exponent $\kappa=1 / 2, \underline{14}-18,21$ that is agreed to be correct to all orders of perturbation theory. At first, this difference was believed to be due to the strong disorder limit applied in Ref. (20). The same result $\kappa=2 / 3$ was obtained, however, also in the limit of weak disorder 22 for a model of non-interacting particles moving on a square lattice via random nearest neighbor hopping in the presence of a $\pi$-flux phase $\underline{23}$ Very recently, it has been pointed out that non-perturbative effects related to topologically non-trivial excitations have to be taken into account in field-theoretical investigations of quantum phase transitions in disordered systems belonging to the chiral symmetry classes $\underline{\underline{24}}$

Besides the divergence of the density of states discussed above, a corresponding divergence of the localization length with $\kappa=1 / 2$, also predicted by theory 21 to occur in a very narrow energy interval close to $E=0$, has been successfully observed for a chU model in a numerical study of the smallest Lyapunov exponent only recently despite several previous attempts $\underline{26}-\underline{30}$ Scaling properties of $2 \mathrm{D}$ chiral systems can be studied numerically by the analysis of the length and disorder or energy dependence of Lyapunov exponents defined for quasi-one-dimensional (q1D) systems. In the numerical analysis, strips of finite width $L$ and length $L_{z} \gg L$ are calculated by the transfer matrix method. The quantity of interest is the 
smallest eigenvalue $x_{1}$ of the matrix $\ln \left(T^{\dagger} T\right)$, where $T$ is the transfer matrix. Following the finite size scaling method, from which the localization length of the infinite $2 \mathrm{D}$ system can be inferred by studying q1D samples, we assume that the smallest positive Lyapunov exponent $\gamma=\lim _{L_{z} \rightarrow \infty} x_{1} L / L_{z}$ is a function of the ratio $\ln L / \ln \xi$, where $\xi(E)$ is the correlation length.

Using a bricklayer lattice model with uncorrelated ran-

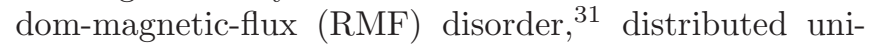
formly with zero mean, a scaling of the dimensionless quantity $z_{1}=x_{1} L / L_{z}$ was observed,,$\underline{25}$

$$
z_{1}(E, L)=F_{1}\left(\frac{\ln L}{\ln \xi(E)}\right)
$$

with the scaling function $F_{1}$ depending only on the ratio $(\ln L) /(\ln \xi(E))$. This model belongs to the chiral unitary symmetry class (broken time-reversal symmetry). The same exponent was found also for a similar model defined on the square lattice $\stackrel{25}{\underline{2}}$ This result confirms theoretical predictions that for $|E| \ll E_{0}$ the localization length is $\xi(E)=\xi_{0} \exp \left[A\left(\ln \left(E_{0} /|E|\right)\right)^{\kappa}\right]$ with a universal exponent $\kappa=1 / 2 \stackrel{15,21}{=}$ However, the recent suggestion ${ }^{24}$ that nonperturbative effects have to be taken into account applies also to these investigations.

It is the aim of our work to improve the understanding of critical properties at chiral quantum critical points, and to find possible reasons for the differing critical exponents obtained in the energy dependence of the DOS and in the divergence of the localization length. Therefore, we present in this paper further comprehensive numerical results obtained for different disorder strengths in model systems exhibiting chiral orthogonal, chiral symplectic, or chiral unitary symmetry in the presence of a very strong perpendicular magnetic field. The latter situation is quite intriguing because it is well known that in a 'normal' two-dimensional (diagonal) disordered system with broken time-reversal symmetry (unitary) all electronic states are localized. However, this situation converts into the quantum Hall case, where current carrying critical states appear in the disorder broadened Landau bands, if a strong normal magnetic field is applied. Therefore, the question arises whether in the case of chiral unitary symmetry the properties in the vicinity of the chiral critical point at $E=0$ are also influenced by the presence of a strong magnetic field.

We find in all three cases mentioned above a divergence in the energy dependence of the localization length at $E=0$ with a critical exponent $\kappa \simeq 2 / 3$ similar to the exponent found in Refs. (20,22) for the diverging disorder averaged DOS. This value is, however, in contrast to our previous result for the chiral unitary case without a constant magnetic field, where $\kappa \simeq 1 / 2$ was obtained. We argue that a broken particle-hole symmetry may be responsible for this difference.

\section{MODELS}

We consider two dimensional (2D) tight binding models defined on bi-partite square or bricklayer lattices with periodic boundary conditions applied in both directions. The latter model has recently been shown to be well suited for describing the electronic properties of noninteracting particles on graphene's honeycomb lattice in the presence of disorder 31 Also, in contrast to the square lattice, the van Hove singularities do not coincide with the quantum critical point at $E=0$ which facilitates the observation of the energy dependence of the critical divergences. In the absence of diagonal disorder the Hamiltonian reads

$$
\mathcal{H}=\sum_{\left\langle n \neq n^{\prime}\right\rangle} t_{n n^{\prime}} c_{n}^{\dagger} c_{n^{\prime}}
$$

where $c_{n}^{\dagger}$ and $c_{n}$ are the fermionic creation and annihilation operators at lattice site $n$, and the sum runs over nearest neighbors only. The disorder is incorporated in the real random hopping $(\mathrm{RRH})$ terms

$$
t_{y}=t_{0} \exp \frac{W}{t_{0}} \varepsilon
$$

pointing in the transversal direction and connecting every other pair of atoms (those bonds between the zigzag lines on a hexagonal lattice) in the bricklayer situation. Here, $\{\varepsilon\}$ is a set of uncorrelated random numbers with box probability distribution, $|\varepsilon| \leq 1 / 2$. In our investigation, the strength of the disorder is varied between $W / t_{0}=2$ and $W / t_{0}=8$. The spectral bandwidth $\Delta$ is determined by the sum over the mean of the hopping terms and the coordination number $Z, \Delta=(Z / 2)\left(\left\langle t_{x}\right\rangle+\left\langle t_{y}\right\rangle\right)$, with $t_{x} / t_{0}=1$ and

$$
\left\langle t_{y}\right\rangle=t_{0} \int_{-1 / 2}^{+1 / 2} d \varepsilon \exp \left[\frac{W}{t_{0}} \varepsilon\right]=\frac{2 t_{0}^{2}}{W} \sinh \frac{W}{2 t_{0}} .
$$

$\Delta$ increases by a factor of 6 when the disorder strength $W$ is increased from $W / t_{0}=4$ to $W / t_{0}=8$.

The same off-diagonal disorder type with $W / t_{0}=4$ was used in the chiral symplectic model defined on an ordinary square lattice. We consider a chiral version

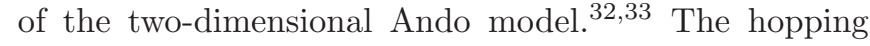
terms $t_{n n^{\prime}}$ are now $2 \times 2$ matrices

$$
t_{\|}=t_{0}\left(\begin{array}{ll}
r & -s \\
-s & r
\end{array}\right), \quad t_{\perp}=t_{y}\left(\begin{array}{ll}
r & i s \\
-i s & r
\end{array}\right)
$$

where $r^{2}+s^{2}=1, s=1 / 2$, and the disorder in the hopping $t_{y}$ is given by Eq. (3).

The chiral unitary model is studied again on a brick-

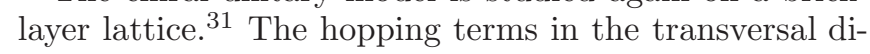
rection are defined as

$$
t_{y}=t_{0} e^{i \theta_{x, y ; x, y \pm a}}
$$


where the phases $\theta_{x, y ; x, y+a}=\theta_{x+2 a, y ; x+2 a, y+a}-\frac{2 \pi e}{h} \Phi_{x, y}$ are determined by the total flux threading the plaquette at $(x, y)$

$$
\Phi_{x, y}=\frac{p}{q} \frac{h}{e}+\phi_{x, y},
$$

where $p / q$ ( $p$ and $q$ are mutually prime integers) is the rational number of magnetic flux quanta $h / e$ per plaquette $2 a^{2}$, and $B=p h /\left(2 q e a^{2}\right)$ is the magnetic flux density perpendicular to the two-dimensional lattice. This differs from the random flux model of our previous work ${ }^{25}$, where the constant magnetic field part was absent. The random part originates from the magnetic fluxes $\phi_{x, y}$, which are uniformly distributed $-f / 2 \leq \phi_{x, y} \leq f / 2$ with zero mean and disorder strength $0 \leq f /(h / e) \leq 1$.

\section{METHOD}

We calculate the two smallest dimensionless scaling variables $z_{1}, z_{2}$ for quasi-one-dimensional samples having an even width $L$ in the range $8 \leq L / a \leq 160$ lattice spacings $a$ using the transfer-matrix-method $\stackrel{34}{ }$ For $E=0$, $z_{1}=2 L / \lambda$ is related to the electronic localization length $\lambda(E=0, L)$. The values of $z_{1}, z_{2}$ were obtained with the relative accuracy $\sqrt{\sigma_{1}} / z_{1} \sim \alpha \times 10^{-4}$, where $\alpha=3$ for small $L$, and $\alpha$ increases to 10 for $L / a=128$ and larger. For zero energy, we checked that the difference

$$
z_{1}(E=0, L)-z_{2}(E=0, L)
$$

is smaller than the numerical uncertainty of our data for all $L$. For the energy dependence, a narrow interval of $|E| \leq 10^{-8} t_{0}$ around the critical point at $E=0$ was considered.

While investigating various disorder strengths increasing from $W / t_{0}=2$ to $W / t_{0}=8$ for the chiral orthogonal model, it turned out that the scaling is more difficult to observe for weak disorder. The reason is that in this case the smallest scaling variable $z_{1}$ is less than 1 , which means that the related localization length exceeds the sample width. Therefore, putting, for instance, $W / t_{0}=2$, we could not observe the insulating regime for non-zero energies even on length scales $L / a \leq 160$, and our data suffer from strong finite-size effects. Therefore, in what follows we discuss only data for stronger disorder $W / t_{0} \geq 5$ with $z_{1} \geq 1$.

We fit our numerical data to the polynomial functions

$$
Z_{1,2}(E, L)=z_{1,2}(E=0, L) \pm \sum_{n}^{n_{f}} b_{n} \chi^{n}
$$

where $z_{1}(E=0, L)$ and $z_{2}(E=0, L)$ are the numerically obtained values related to the two smallest Lyapunov exponents at zero energy. The dimensionless scaling parameter $\chi$ is defined as

$$
\chi=\frac{[\ln (L) / A]^{x_{3}}}{\left[\ln \left(E_{0} /|E|\right)\right]^{x_{2}}} .
$$

We assume the following energy dependence of the correlation length

$$
\ln \left(\xi(E) / \xi_{0}\right)=A\left|\ln \left(E_{0} /|E|\right)\right|^{\kappa},
$$

with unknown parameters $E_{0}, \xi_{0}$, and critical exponent $\kappa=x_{2} / x_{3}$.

The total number of fitting parameters is $N_{p}=4+$ $n_{f}$, where $n_{f}$ determines the highest order of polynomial in Eq. (91). The additional four parameters are the two exponents $x_{2}$ and $x_{3}$, the energy $E_{0}$, and the length scale $\xi_{0}$. The latter was fixed to $\xi_{0}=1$ in all our analysis $\underline{42}$ In the fitting procedure, we minimize the following function

$$
\begin{aligned}
F & =\frac{1}{N} \sum_{n}^{N}\left(\frac{\left[z_{1}^{(n)}(E, L)-Z_{1}(E, L)\right]^{2}}{\sigma_{1}(E, L)}\right. \\
& \left.+\frac{\left[z_{2}^{(n)}(E, L)-Z_{2}(E, L)\right]^{2}}{\sigma_{2}(E, L)}\right) .
\end{aligned}
$$

In Eq. (12), $z_{1}^{(n)}$ and $z_{2}^{(n)}$ are the numerically obtained data for the first and the second smallest scaling variable, and $\sigma_{1}$ and $\sigma_{2}$ are their numerical uncertainties. To estimate the accuracy of the fit, we create a statistical ensemble of up to $N_{\text {stat }}=50$ sets with initial conditions taken as $z_{1}+\sqrt{\sigma_{1}} \times \epsilon$ with random number $|\epsilon|<1$.

After fitting the obtained numerical data for $z_{1}$ and $z_{2}$ to the function (91), we change the number of fitting parameters (i.e., the order $n_{f}$ of the polynomial) in order to check the reliability of the fit. Although we do not expect strong finite size effects, we also vary the smallest size of the systems taken into account in the data ensembles. Another check of the stability of the resulting exponent is to reduce the energy interval, for instance to values $E<10^{-13} t_{0}$.

\section{RESULTS}

The results for all models analyzed are summarized in Table I. For the chiral orthogonal model, we present data for four different values of the disorder strength in the range $5 \leq W / t_{0} \leq 8$. These disorders are strong enough to assure that the smallest scaling variable is $z_{1}>$ 1 , so that we are in the localized regime for non-zero energies. The exponents $\kappa$ are close to the value $2 / 3$. Small deviations are seen for weak disorder $W=5$.

For the chiral symplectic system, we analyzed only one value of the disorder strength, $W / t_{0}=4$. The obtained value for the critical exponent is compatible with $\kappa=$ $2 / 3$ and agrees well with the one found for the chiral orthogonal symmetry.

For chiral unitary systems, we consider a model with a constant magnetic flux density $B=(1 / 12) h /\left(2 e a^{2}\right)$ and $\mathrm{RMF}$ disorder strength $f /(h / e)=0.5$ (labeled chU[1/12, $0.5])$, and also a model where the constant magnetic field $B=(1 / 16) h /\left(2 e a^{2}\right)$ is combined with real hopping terms $t_{y}$ as given by Eq. (3) (model chU[1/16, 5.0]). Both systems exhibit a critical exponent consistent with a value 
TABLE I: The critical parameters for models with RRH or RMF disorder with a strong constant magnetic field as obtained by the fitting procedure described in Section III In the ensembles marked by ${ }^{*}$, a restricted energy interval $|E|<10^{-13} t_{0}$ was used instead of $|E|<10^{-8} t_{0}$. The labeling of the chiral orthogonal chO[ ], symplectic chS[ ] and unitary models chU[ ] is explained in the text below. These models exhibit a critical exponent close to $2 / 3$.

\begin{tabular}{|c|c|c|c|c|c|}
\hline$N_{p}$ & $L / a$ & $\ln E_{0}$ & $\kappa$ & $x_{3}$ & $\frac{F_{\min }}{2}$ \\
\hline \multicolumn{6}{|c|}{ chO: RRH $W / t_{0}=5, z_{1}=1.35$} \\
\hline 5 & $12-96$ & $-1.60 \pm 0.41$ & $0.813 \pm 0.013$ & $1.100 \pm 0.03$ & 0.29 \\
\hline $5^{*}$ & $12-96$ & $-2.3 \pm 1.5$ & $0.801 \pm 0.03$ & $1.17 \pm 0.02$ & 0.11 \\
\hline \multicolumn{6}{|c|}{$\operatorname{chO}:$ RRH $W / t_{0}=6, z_{1}=1.79$} \\
\hline 5 & $12-160$ & $-5.50 \pm 0.20$ & $0.665 \pm 0.006$ & $1.220 \pm 0.003$ & 2.24 \\
\hline $5^{*}$ & $24-160$ & $-4.17 \pm 2.02$ & $0.724 \pm 0.046$ & $1.160 \pm 0.11$ & 0.28 \\
\hline \multicolumn{6}{|c|}{$\operatorname{chO}:$ RRH $W / t_{0}=7, z_{1}=2.23$} \\
\hline 5 & $12-128$ & $-3.65 \pm 0.18$ & $0.675 \pm 0.005$ & $1.272 \pm 0.002$ & 4.89 \\
\hline $5^{*}$ & $12-128$ & $-4.4 \pm 1.5$ & $0.693 \pm 0.03$ & $1.204 \pm 0.02$ & 0.39 \\
\hline \multicolumn{6}{|c|}{ chO: $\mathrm{RRH} W / t_{0}=8, z_{1}=2.65$} \\
\hline $5^{*}$ & $12-96$ & $-6.4 \pm 0.5$ & $0.628 \pm 0.01$ & $1.25 \pm 0.03$ & 1.71 \\
\hline \multicolumn{6}{|c|}{ chS: RRH $W / t_{0}=4, z_{1}=1.97$} \\
\hline 4 & $8-160$ & $-5.4 \pm 0.1$ & $0.687 \pm 0.049$ & $1.403 \pm 0.021$ & 7.40 \\
\hline $4^{*}$ & $8-160$ & $-7.5 \pm 0.5$ & $0.692 \pm 0.015$ & $1.257 \pm 0.004$ & 1.22 \\
\hline 4 & $16-160$ & $-6.1 \pm 0.1$ & $0.676 \pm 0.058$ & $1.409 \pm 0.041$ & 6.40 \\
\hline 5 & $16-160$ & $-3.4 \pm 0.1$ & $0.796 \pm 0.078$ & $1.045 \pm 0.033$ & 2.83 \\
\hline 5 & $48-160$ & $-6.1 \pm 0.7$ & $0.664 \pm 0.028$ & $1.186 \pm 0.025$ & 0.98 \\
\hline \multirow{2}{*}{\multicolumn{6}{|c|}{$\operatorname{chU}[1 / 16,5.0]: B=1 / 16 h /\left(2 e a^{2}\right), \mathrm{RRH} W / t_{0}=5}}$, \\
\hline & & & & & \\
\hline 6 & $32-96$ & $-1.74 \pm 0.37$ & $0.636 \pm 0.01$ & $1.27 \pm 0.13$ & 1.4 \\
\hline 7 & $32-96$ & $-1.23 \pm 0.64$ & $0.648 \pm 0.02$ & $1.17 \pm 0.17$ & 0.8 \\
\hline \multicolumn{6}{|c|}{$\begin{array}{r}\operatorname{chU}[1 / 12,0.5]: B=1 / 12 h /\left(2 e a^{2}\right), \operatorname{RMF} f=0.5 h / e, \\
z_{1}=1.03\end{array}$} \\
\hline 4 & $24-72$ & $-6.74 \pm 0.24$ & $0.567 \pm 0.008$ & $1.75 \pm 0.003$ & 3.504 \\
\hline 5 & $24-72$ & $-4.14 \pm 0.65$ & $0.643 \pm 0.02$ & $1.313 \pm 0.02$ & 2.142 \\
\hline 6 & $24-72$ & $-3.45 \pm 0.65$ & $0.663 \pm 0.02$ & $1.332 \pm 0.23$ & 2.055 \\
\hline 7 & $24-72$ & $-3.02 \pm 0.96$ & $0.676 \pm 0.03$ & $1.411 \pm 0.33$ & 2.016 \\
\hline
\end{tabular}

$2 / 3$. Please note that the energies of the quantum Hall critical states belonging to the zeroth Landau band are split $\underline{31}$ by the disorder, and so they do not interfere with the chiral critical point at $E=0$ studied here.

To compare the new results with our previous data published in Ref. 25 for the chiral unitary system without a constant magnetic field, we use the present fitting method also for the analysis of our previous data. In Table II we see that the critical exponent for the model $\operatorname{chU}[0,0.5]$ is consistent with the value $1 / 2$ as reported previously, 25 but differs significantly from the value $2 / 3$ obtained here for all other ensembles. Also, in order to check a possible disorder dependence of $\kappa$, again a bricklayer model with zero constant magnetic field but with a stronger RMF disorder strength $f=0.7 \mathrm{~h} / \mathrm{e}$ (la-
TABLE II: The critical parameters for models with RMF disorder and zero or small constant magnetic fields as obtained by the fitting procedure described in Section [II A critical exponent close to $1 / 2$ is found. The energy interval used was $|E|<10^{-10} t_{0}$. The labeling of the various models chU[ ] is explained in the text.

\begin{tabular}{|c|c|c|c|c|c|}
\hline$N_{p}$ & $L / a$ & $\ln E_{0}$ & $\kappa$ & $x_{3}$ & $\frac{F_{\min }}{2}$ \\
\hline \multicolumn{6}{|c|}{ 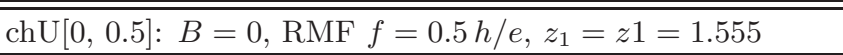 } \\
\hline 6 & $8-160$ & $-2.6 \pm 1.6$ & $0.414 \pm 0.04$ & $2.49 \pm 0.44$ & 0.91 \\
\hline 7 & $8-160$ & $0.25 \pm 2.5$ & & & 1.43 \\
\hline 8 & $8-160$ & $1.86 \pm 1.1$ & $0.511 \pm 0.025$ & & 1.28 \\
\hline \multicolumn{6}{|c|}{$\operatorname{chU}[0,0.7]: B=0, \mathrm{RMF} f=0.7 \mathrm{~h} / e, z_{1}=1.22$} \\
\hline 5 & $24-96$ & $-4.5 \pm 1.5$ & $0.515 \pm 0.04$ & $1.86 \pm 0.20$ & 2.43 \\
\hline 5 & $32-96$ & -3.5 & 0.55 & 1 & 2.01 \\
\hline 5 & $40-96$ & $-4.0 \pm 1.7$ & $0.54 \pm 0.04$ & $1.65 \pm 0.10$ & 1.86 \\
\hline \multicolumn{6}{|c|}{$\operatorname{chU}[0$, as $]: B=0, \operatorname{RMF} f /(h / e)=-0.25,0.125, z_{1}=1.328$} \\
\hline 5 & $16-96$ & $-4.58 \pm 1.8$ & $0.474 \pm 0.04$ & $2.156 \pm 0.02$ & 1.36 \\
\hline 6 & $16-96$ & $-1.66 \pm 2.6$ & $0.535 \pm 0.05$ & $2.40 \pm 0.38$ & 1.22 \\
\hline \multicolumn{6}{|c|}{$\begin{array}{r}\operatorname{chU}[1 / 10 \mathrm{~K}, 0.5]: B=1 / 10000 h /\left(2 e a^{2}\right), \mathrm{RMF} f=0.5 \mathrm{~h} / e, \\
z_{1}=1.5559\end{array}$} \\
\hline 7 & $24-96$ & $0.38 \pm 4$ & $0.464 \pm 0.07$ & $2.38 \pm 1.14$ & 2.01 \\
\hline 8 & $24-96$ & $1 \pm 1$ & $0.475 \pm 0.02$ & $3.16 \pm 0.75$ & 2.39 \\
\hline \multicolumn{6}{|c|}{$\begin{array}{r}\operatorname{chU}[1 / 1 \mathrm{~K}, 0.5]: B=1 / 1000 h /\left(2 e a^{2}\right), \operatorname{RMF} f=0.5 h / e, \\
z_{1}=1.557\end{array}$} \\
\hline 6 & $24-96$ & $-0.7 \pm 3.7$ & $0.448 \pm 0.063$ & $2.27 \pm 0.64$ & 1.82 \\
\hline 7 & $24-96$ & $2.7 \pm 2.5$ & $0.511 \pm 0.043$ & $2.40 \pm 0.75$ & 2.52 \\
\hline 8 & $24-96$ & $2.8 \pm 1.4$ & $0.512 \pm 0.023$ & $2.73 \pm 0.44$ & 2.22 \\
\hline
\end{tabular}

beled $\operatorname{chU}[0,0.7])$ was calculated. We obtain the same value $\kappa \simeq 1 / 2$ and so confirming our previous results. In Fig. 1 we compare some of the raw numerical data for $z_{i}(E, L)-z_{i}(E=0, L), i=1,2$, with the fit given by Eq. (9). Some other models, listed in Table II which also exhibit an exponent $\kappa=1 / 2$ will be discussed in the next section.

\section{DISCUSSION AND CONCLUSIONS}

Our numerical data for two-dimensional models with various chiral symmetry confirm the logarithmic energy dependence of the localization length reported previously $\stackrel{25}{=}$ Here, we find that this logarithmic dependence is determined by the critical exponent $\kappa$, which is universal and close to $2 / 3$ for chO, chS, and chU (with magnetic field) symmetry classes. The only exception is the chiral unitary system with zero constant magnetic field where $\kappa \simeq 1 / 2$. We have confirmed this previous result by additional calculations for a RMF disorder strength $f=0.7 \mathrm{~h} / e$. Therefore, the chiral unitary models with and without constant magnetic field studied here exhibit different critical behaviors.

One source for this outcome could be that the distri- 


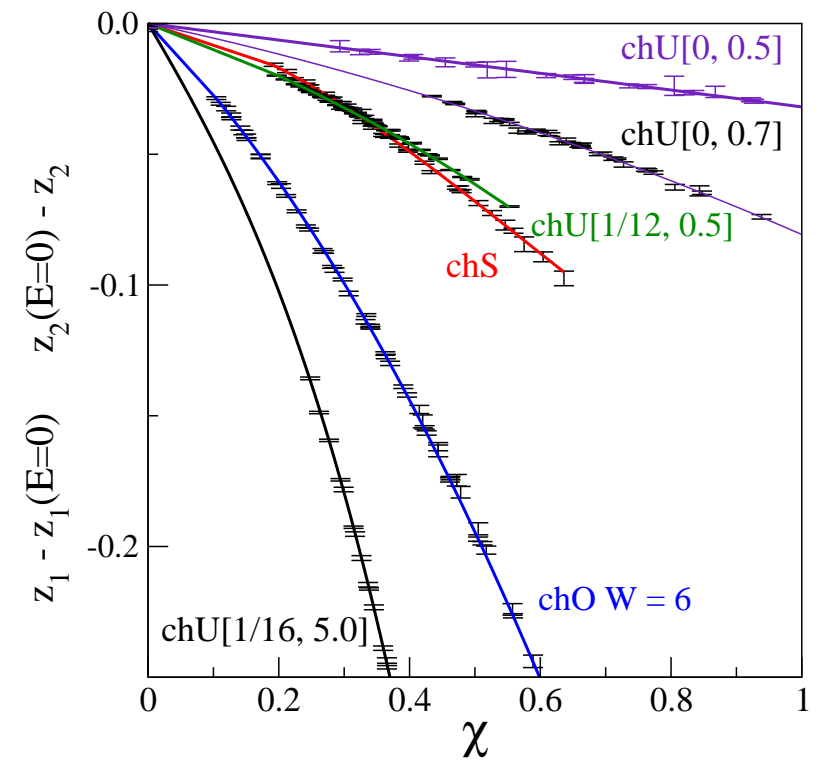

FIG. 1: (Color online) The difference $-\mid z_{i}(E, L)-z_{i}(E=$ $0, L) \mid$ of the two smallest dimensionless scaling variables plotted as a function of the parameter $\chi$ with corresponding fits (solid lines) for some systems listed in Table [ and II The previously obtained chU $[0,0.5]$ data $^{25}$ are shown only in part since $\chi$ increases up to values of $\sim 10$ in this case.

bution of the random magnetic fluxes, which was chosen to be symmetric about zero flux in the $B=0$ situation, turns into an asymmetric one due to the addition of a spatially constant magnetic flux in the finite magnetic field case. We have checked that an asymmetric distribution of RMF-fluxes still leads to a critical exponent $1 / 2$ as long as the average magnetic flux remains zero. In these calculations, the random flux was taken to be $-0.25 \mathrm{~h} / \mathrm{e}$ with probability $1 / 3$ and $0.125 \mathrm{~h} / \mathrm{e}$ with probability $2 / 3$ so that the distribution of random fluxes is asymmetric albeit the mean value is zero. Using a 6-parametric fit as given by Eq. (10), we obtained that $\kappa=0.535 \pm 0.05$ for $16 \leq L / a \leq 96$. The details for the antisymmetric RMF distribution chU[0, as] can be seen in Table II]

Another reason for the different exponents could be that, for the largest system size studied, the disorder strength is still too small in the zero constant magnetic field situation. Due to the periodicity, the effect of the random flux disorder $-f / 2 \leq \phi_{x, y} \leq f / 2$ appearing in the phases $\exp \left(-i \theta_{x, y+a ; x, y}\right)$ with $\theta_{x, y+a ; x, y}=$ $\theta_{x+2 a, y ; x+2 a, y+a}-2 \pi e \phi_{x, y} / h$ is bounded, being maximal for disorder strength $f=1.0 \mathrm{~h} / \mathrm{e}$ for the bricklayer lattice. The dependence of the smallest scaling variable $z_{1}(E=0, f)$ on the disorder strength $f \geq 0.01$ is shown in Fig. (2) for $B=0, p / q=1 / 24$, and $p / q=1 / 12$, respectively. In the small disorder regime, the behavior is complicated and not important for the problem of different critical exponents discussed here. For large $f$, the $z_{1}(E=0, f)$ decrease in all RMF models, independent of the presence or absence of a constant magnetic field, and become equal $\sim 0.76$ for $f=h / e$. Usually, a

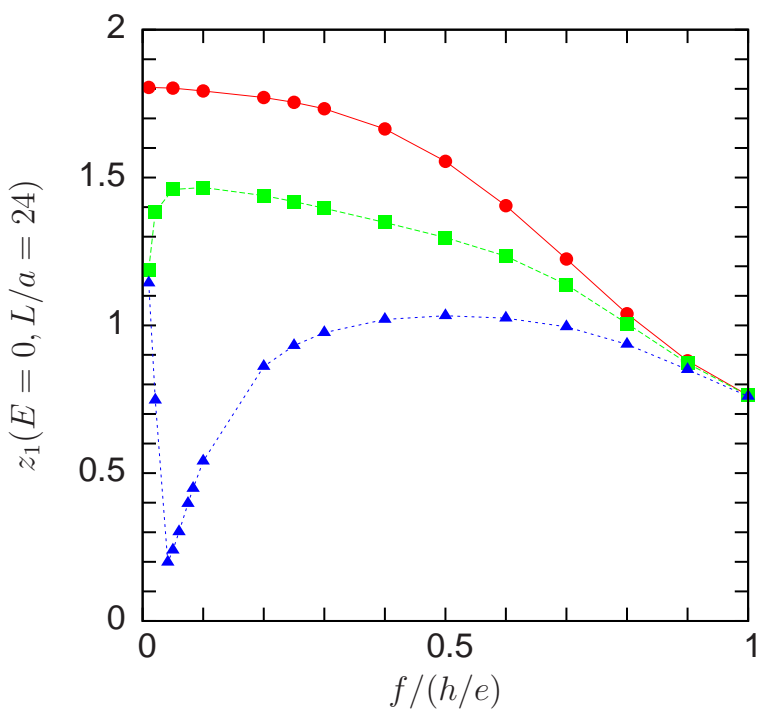

FIG. 2: (Color online) The smallest scaling variable $z_{1}(E=$ $0, f)$ versus RMF disorder strength $f$. The constant magnetic field is zero (red dots), $B=1 / 24 \times h /\left(2 e a^{2}\right)$ (green squares), and $B=1 / 12 \times h /\left(2 e a^{2}\right)$ (blue triangles), respectively. These results do not dependent on the system width in the range $24 \leq L / a \leq 128$ checked.

decreasing $z_{1}$ is equivalent to an increasing localization length $\lambda=2 L / z_{1}$. A similar increase of the two-terminal conductance with increasing RMF-disorder strength has been reported previously $\frac{35}{3}$ The behavior of the RMFmodels is opposite to the chO and chS situation where $z_{1}(E=0, W)$ becomes larger with increasing disorder strength $W$. However, when comparing the various values of $z_{1}(E=0)$ obtained for different models (see Table I), which are related to the effective disorder strength, the above suggestion that too weak a disorder was applied in the RMF-model without $B$-field seems to be unlikely, and we do not believe that this is the correct explanation for the observed difference in the critical exponents.

Another distinction between the two chU cases is the behavior of the density of states $\rho(E)$ very close to $E=0$. Although both the chU models with and without strong magnetic field exhibit a narrow depression,, 36 with $\rho(E)$ going to zero at the critical point, the disorder dependence is quite different. With increasing randommagnetic-flux disorder strength, the energy range of the depression gets narrower in the absence of a constant $B$ while it increases when a strong perpendicular magnetic field is present. The appearance of such 'microgaps' has been attributed to the non-perturbative ergodic regime $17,37,38$ of the chiral model, i.e., the localization length greatly exceeds the sample size so that for time scales large compared to the diffusion time the particle extends over the whole sample. A similar behavior was observed also for the chS model $\stackrel{39}{\underline{ }}$ We note, however, that DOS depressions ('microgaps') are absent in our numerical calculations performed for the chO case. For strong disorder, a narrow peak appears instead at $E=0, \stackrel{39}{\underline{3}}$ pre- 
sumably originating from the different type of disorder (real random hopping) that in contrast to the complex random phases in the RMF models can lead to isolated sites having eigenenergies around $E=0$. This extra peak is observed for both the bricklayer and the square lattice systems. Nevertheless, it is unclear whether the different disorder dependence of the DOS around the critical point can account for the observed difference in the critical exponents.

Finally, we would like to remind the reader that the presence of a strong magnetic field causes a special topological term in the appropriate field theories $\frac{3,40,41}{4 h}$ This extra term is responsible for the occurrence of current carrying states that are essential for the explanation of the integer quantum Hall effect, and so it accounts for the difference between the ordinary Gaussian unitary ensemble and the quantum Hall situation, i.e., the appearance of critical electronic states in the latter case.

Furthermore, the strong magnetic field may effectively restore the particle-hole symmetry that is usually lacking when time-reversal symmetry is broken. The recovery of the particle-hole symmetry can take place when the scattering between electronic states belonging to different disorder broadened Landau bands is suppressed by a large energy separation. Thus, a single Landau band model containing a chiral quantum critical point at $E=0$ is effectively created in the chiral unitary case with an additional very strong, spatially constant, perpendicular magnetic field. We therefore suggest that the presence or absence of particle-hole symmetry is responsible for the different critical exponents found in our numerical calculations of various chiral unitary models. To check this view, we investigated also chiral unitary models with weaker constant magnetic fields $p / q=1 / 10000$ and $p / q=1 / 1000$ (see $\operatorname{chU}[1 / 10 \mathrm{~K}, 0.5]$ and $\operatorname{chU}[1 / 1 \mathrm{~K}$, $0.5]$ in Table III) and found critical exponents $\simeq 1 / 2$ as in the magnetic field free case.

In conclusion, we calculated the two smallest Lyapunov exponents of lattice models belonging to the chiral orthogonal, chiral symplectic, and to the chiral unitary class in the presence of a strong spatially constant magnetic field. We found in all cases a critical exponent $\kappa \simeq 2 / 3$ that governs the divergence of the localization length at the band center $E=0$. This result is in agreement with previous calculations for the diverging energy dependence of the density of states $\underline{20,22}$ It is, however, at variance with the earlier analytically obtained results $\stackrel{15,21}{ }$ While reasons for this difference were suggested in Refs. (1, 20,22, 24), the origin of the numerically 25 obtained value $\kappa \simeq 1 / 2$ for chiral unitary lattice models in the absence of a strong perpendicular magnetic field is still an open question. The latter result was again corroborated in the present study and suggested to be due to the absence of particle-hole symmetry in the RMF-model without a strong spatially constant magnetic field. However, due to the limited achievable system size in the numerical calculations, a magnetic field induced transition from the ballistic to the diffusive regime as discussed above, cannot be completely excluded.

\section{Acknowledgments}

P. M. thanks Project VEGA 0633/09 for financial support.
1 F. Evers and A. D. Mirlin, Rev. Mod. Phys. 80, 1355 (2008).

2 F. Wegner, Z. Physi, B 35, 207 (1979).

3 A. M. M. Pruisken, Nucl. Phys. B 235, 277 (1984).

4 X.-L. Qi and S.-C. Zhang, Rev. Mod. Phys. 83, 1057 (2011).

${ }^{5}$ K. S. Novoselov, A. Geim, S. V. Mozorov, D. Jiang, M. I. Katsnelson, I. V. Grigorieva, S. V. Dubonos, and A. A. Firsov, Nature 438, 197 (2005).

6 Y. Zhang, Y.-W. Tan, H. L. Stormer, and P. Kim, Nature 438, 201 (2005).

7 J. J. M. Verbaarschot and I. Zahed, Phys. Rev. Lett. 70, 3852 (1993).

8 A. Altland and M. R. Zirnbauer, Phys. Rev. B 55, 1142 (1997).

9 J. Miller and J. Wang, Phys. Rev. Lett. 76, 1461 (1996).

10 P. W. Brouwer, C. Mudry, B. D. Simons, and A. Altland, Phys. Rev. Lett. 81, 862 (1998).

11 A. Furusaki, Phys. Rev. Lett. 82, 604 (1999).

12 Y. Asada, K. Slevin, and T. Ohtsuki, J. Phys. Soc. Jap. 72, 145 (2003).

13 M. Bocquet and J. T. Chalker, Phys. Rev. B 67, 1 (2003).
14 R. Gade and F. Wegner, Nucl. Phys. B360, 213 (1991).

15 R. Gade, Nucl. Phys. B398, 499 (1993).

16 A. W. W. Ludwig, M. P. A. Fisher, R. Shankar, and G. Grinstein, Phys. Rev. B 50, 7526 (1994).

17 A. Altland and B. D. Simons, Nucl. Phys. B 562, 445 (1999).

18 P. W. Brouwer, C. Mudry, and A. Furusaki, Phys. Rev. Lett. 84, 2913 (2000).

19 S. N. Evangelou and D. E. Katsanos, J. Phys. A: Math. Gen. 36, 3237 (2003).

20 O. Motrunich, K. Damle, and D. A. Huse, Phys. Rev. B 65, 064206 (2002).

21 M. Fabrizio and C. Castelliani, Nucl. Phys. B 583, 542 (2000).

22 C. Mudry, S. Ryu, and A. Furusaki, Phys. Rev. B 67, 064202 (2003).

23 Y. Hatsugai, X.-G. Wen, and M. Kohmoto, Phys. Rev. B 56, 1061 (1997).

24 E. J. König, P. M. Ostrovsky, I. V. Protopopov, and A. D. Mirlin, arXiv:1201.6288v1 (2012).

25 P. Markoš and L. Schweitzer, Phys. Rev. B 81, 205432 (2010). 
26 V. Z. Cerovski, Phys. Rev. B 62, 12775 (2000).

27 A. Eilmes, R. A. Römer, and M. Schreiber, Physica B 296 , 46 (2001).

28 V. Z. Cerovski, Phys. Rev. B 64, 161101(R) (2001).

29 A. Eilmes and R. A. Römer, phys. stat. sol. (b) 241, 2079 (2004).

30 P. Markoš and L. Schweitzer, Phys. Rev. B 76, 115318 (2007).

31 L. Schweitzer and P. Markoš, Phys. Rev. B 78, 205419 (2008).

32 T. Ando, Phys. Rev. B 40, 5325 (1989).

33 P. Markoš and L. Schweitzer, J. Phys. A 39, 3221 (2006).

34 J. B. Pendry, A. MacKinnon, and P. J. Roberts, Proc. R. Soc. London A 437, 67 (1992).

35 L. Schweitzer and P. Markoš, Physica E 40, 1335 (2008).
36 L. Schweitzer, Phys. Rev. B 80, 245430 (2009).

37 B. D. Simons and A. Altland, in Theoretical Physics at the End of the XXth Century, edited by Y. Saint-Aubin and L. Vinet (Springer, 2001), CRM Series in Mathematical Physics, pp. 451-558.

38 A. Altland and R. Merkt, Nucl. Phys. B 607, 511 (2001).

39 P. Markoš and L. Schweitzer, Physica B in press (2012).

${ }^{40}$ H. Levine, S. B. Libby, and A. M. M. Pruisken, Phys. Rev. Lett. 51, 1915 (1983).

41 H. A. Weidenmüller, Nucl. Phys. B 290, 87 (1987).

42 The analysis shows that the parameter $\xi_{0}$ is not independent of other fitting parameters. Changes of $\xi_{0}$ are compensated by corresponding changes of other parameters, but do not influence the value of the exponent $\kappa$. 